Image Processing, Geoinformatics and Information Security

\title{
ON A MOMENT PROBLEM FOR SETS OF POINTS IN THE COMPLEX PLANE
}

\author{
V.P. Tsvetov \\ Samara National Research University, Samara, Russia
}

\begin{abstract}
This paper deals with a uniqueness problem of determination a set of points in the complex plane by the degree-like moments. We discuss applications of these results to determine the similarity of flat polygonal lines in contour analysis method.
\end{abstract}

Keywords: moment problem, contour analysis, image processing.

Citation: Tsvetov VP. On a moment problem for sets of points in the complex plane. CEUR Workshop Proceedings, 2016; 1638: 411-418. DOI: 10.18287/1613-0073-2016-1638-411-418

\section{Introduction}

A moment problem is closely associated with many questions of functional analysis [1], integral geometry [2,3], problems of interpolations, and function classifications [4]. Several problems concerning the uniqueness of solutions of operator equations can be reformulated in terms of determining continuous linear functional $f: X \rightarrow \mathrm{C}$ by the known values on a system of basic elements $\left\{z_{m}\right\}_{m \in I \subseteq N_{0}}$ of a normed linear space $X$ :

$f\left(z_{m}\right)=s_{m}$.

In applications $X$ is a space of complex-valued functions that are continuous on compact set $K$. Such space with the uniform norm is denoted as $C(K)$. By $C(K)^{*}$ we denote the linear space that topologically conjugates to $C(K)$. So $C(K)^{*}$ contains all continuous linear functionals $f: C(K) \rightarrow \mathrm{C}$. Based on the Riesz-Radon theorem we know that $C(K)^{*}$ is isometric to the space of Radon measures with compact support on $K[1,5]$. Hence any linear functional can be uniquely represented in the form

$$
f(z)=\int_{K} z(t) d \mu_{f}(t)
$$


here $\mu_{f}$ is a measure with compact support $K_{f} \subseteq K$ that is uniquely defined by the functional $f$.

In this paper we will be interested in the case of (1) or (2), where $K \subset \mathrm{R}^{2}$, $z_{m}(t)=z_{m}(x, y)=z^{m}=(x+i y)^{m}, \quad i^{2}=-1$, and $\mu_{f}(t)$ is a function of bounded variation with compact support $K_{f} \subseteq K \subset \mathrm{R}^{2}$. The integral in (2) is understood as an integral of Lebesgue-Stieltjes.

If $K_{f}=\left\{t_{j} \mid t_{j}=\left(x_{j}, y_{j}\right), j \in 1 . . k\right\}$ is a finite set of points, then the integral in (2) is reduced to the finite sum

$$
f(z)=\sum_{j=1}^{k} z\left(t_{j}\right) \cdot \mu_{f}\left(t_{j}\right)=\sum_{j=1}^{k} z_{j} \cdot \mu_{j}^{f},
$$

where $z_{j}=x_{j}+i y_{j}$, and $\mu_{j}^{f} \in \mathrm{C}$. So (1) takes one of the forms

$$
f\left(z^{m}\right)=\int_{K \subset \mathrm{R}^{2}} z^{m}(t) d \mu_{f}(t)=\int_{K_{f} \subset \mathrm{R}^{2}} z^{m}(t) d \mu_{f}(t)=S_{m},
$$

or

$$
f\left(z^{m}\right)=\sum_{j=1}^{k} z_{j}^{m} \cdot \mu_{j}^{f}=s_{m} .
$$

We will show the uniqueness of a linear functional $f$ (and so $K_{f}$ ) that is determined from (5) by a known finite number of values $s_{m}$. Then we extend this result to the special case of (4), when the compact support $K_{f}$ is a polyline with a finite number of segments, and the integral is understood as a line integral along a plane curve.

Note that the moment problem (5) arises in a contour analysis based on the integral representations for Gaussian beams [6].

It is easy to give an example of different compact subsets $K_{f} \subseteq K \subset \mathrm{R}^{2}$, which produce an equal moments $S_{m}$ to a corresponding functionals $f$, even in the case $m \in I=\mathrm{N}_{0}$.

As a compact $K \subset \mathrm{R}^{2}$ we consider a circle $K=\left\{(x, y) \mid x^{2}+y^{2} \leq r_{0}^{2}\right\}$ and define a family of subcompacts:

$$
\begin{aligned}
& K_{1}^{r_{1}}=\left\{(x, y) \mid x^{2}+y^{2}=r_{1}^{2}<r_{0}^{2}\right\}, \\
& K_{2}^{r_{2}, r_{3}}=\left\{(x, y) \mid r_{2}^{2} \leq x^{2}+y^{2} \leq r_{3}^{2}<r_{0}^{2}\right\}, \\
& K_{3}^{r_{4}}=\left\{(x, y) \mid x^{2}+y^{2} \leq r_{4}^{2}<r_{0}^{2}\right\} .
\end{aligned}
$$

Then we define continuous linear functionals

$$
f_{1}^{r_{1}}(z)=\int_{K_{1}^{n_{1}}} z(x, y) d L \in C(K)^{*},
$$




$$
\begin{aligned}
& f_{2}^{r_{2}, r_{3}}(z)=\iint_{K_{2}^{2, r_{3}}} z(x, y) d x d y \in C(K)^{*}, \\
& f_{3}^{r_{4}}(z)=\iint_{K_{1}^{r_{4}}} z(x, y) d x d y \in C(K)^{*},
\end{aligned}
$$

where the integral in (6) is understood as a line integral.

For all $m \in \mathrm{N}_{0}$ functions $z_{m}(x, y)=z^{m}=(x+i y)^{m}$ form an orthogonal system over the scalar products

$$
\begin{aligned}
& \left\langle z_{1}, z_{2}\right\rangle_{1}=\int_{K_{1}^{n}} z_{1}(x, y) \overline{z_{2}(x, y)} d L, \\
& \left\langle z_{1}, z_{2}\right\rangle_{2}=\iint_{K_{2}^{\prime, 2,3}} z_{1}(x, y) \overline{z_{2}(x, y)} d x d y, \\
& \left\langle z_{1}, z_{2}\right\rangle_{3}=\iint_{K_{1}^{r_{4}}} z_{1}(x, y) \overline{z_{2}(x, y)} d x d y,
\end{aligned}
$$

So for all $m>0$ we have the zero moments

$$
\begin{aligned}
& f_{1}^{r_{1}}\left(z^{m}\right)=S_{m}^{1}=\int_{K_{1}^{n_{1}}} z^{m} d L=0, \\
& f_{2}^{r_{2}, r_{3}}\left(z^{m}\right)=S_{m}^{2}=\iint_{K_{2}^{n_{2}, r_{3}}} z^{m} d x d y=0, \\
& f_{3}^{r_{4}}\left(z^{m}\right)=S_{m}^{3}=\iint_{K_{1}^{r_{4}}} z^{m} d x d y=0,
\end{aligned}
$$

and non zero moments $S_{0}^{1}=2 \pi r_{1}, S_{0}^{2}=\pi\left(r_{3}^{2}-r_{2}^{2}\right), S_{0}^{3}=\pi r_{4}^{2}$, for $m=0$.

Then we fix $0<r_{1}<\frac{r_{0}^{2}}{2}$ and set $r_{4}=\sqrt{2 r_{1}}, r_{3}^{2}=r_{4}^{2}+r_{2}^{2}$. It's clear that for all $0<r_{2}^{2}<r_{0}^{2}-2 r_{1}$ we obtain an infinite number of different continuous linear functionals $f_{1}^{r_{1}}, f_{2}^{r_{2}, r_{3}}, f_{3}^{r_{1}}$ with compact supports $K_{1}^{r_{1}}, K_{2}^{r_{2}, r_{3}}, K_{3}^{r_{4}}$, and equal moments $f_{1}^{r_{1}}\left(z^{m}\right), f_{2}^{r_{2}, r_{3}}\left(z^{m}\right), f_{3}^{r_{1}}\left(z^{m}\right)$.

This is explained by the fact that the set of functions $z_{m}(x, y)=z^{m}=(x+i y)^{m}$ is not a dense set in $C(K)$. According to the Weierstrass theorem, a dense set is formed by the system of polynomials $p_{m, n}(x, y)=x^{m} y^{n}, m, n \in \mathrm{N}_{0}$. Based on the representations $x=\operatorname{Re}(z)=\frac{z+\bar{z}}{2}, y=\operatorname{Im}(z)=\frac{z-\bar{z}}{2}$, we can say that the system of functions $P_{m, n}(x, y)=z^{m} \bar{z}^{n}$ has the same quality.

Thus any continuous linear functionals $f \in C(K)^{*}$, including those that are defined by (6)-(8) will be uniquely determined by the system of values 


$$
f\left(z^{m} \bar{z}^{n}\right)=S_{m, n}, m, n \in \mathrm{N}_{0} .
$$

If we consider the linear functionals defined by integrals over plain domains or rectifiable curves, then the system of moments (9) will uniquely determine bounded curve or plain domain.

\section{Moment problem for a finite set of points}

Let's consider a moment problem in the following form. From a given system of equations

$$
\sum_{j=1}^{k} z_{j}^{m} \cdot \mu_{j}=s_{m}, m \in 0 . . M, M \in \mathrm{N}
$$

we want to determine a set of pairs of complex numbers $S=\left\{\left(z_{j}, \mu_{j}\right)\right\}_{j=1}^{k}$ under the assumption that all the numbers $z_{j}$ are pairwise distinct, and $\mu_{j} \neq 0$. In (10) we put $z^{0}=1$ for all $\mathrm{z} \in \mathrm{C}$.

Two sets $S=\left\{\left(z_{j}, \mu_{j}\right)\right\}_{j=1}^{k}$ and $\tilde{S}=\left\{\left(\tilde{z}_{j}, \tilde{\mu}_{j}\right)\right\}_{j=1}^{k}$ will be considered as equivalent $S \approx \tilde{S}$, if there exists a total bijection $h: 1 . . k \rightarrow 1 . . k$ that is $z_{j}=\tilde{z}_{h(j)}$ and $\mu_{j}=\tilde{\mu}_{h(j)}$ (in other words, if they match up to a permutation).

We are interested in the uniqueness of the solution for the problem above, which is understood as equivalent in the above sense.

Suppose that there are two sets $S$ and $\tilde{S}$ that satisfies (10). Therefore we have the following system of equations

$$
\sum_{j=1}^{k}\left(z_{j}^{m} \cdot \mu_{j}-\tilde{z}_{j}^{m} \cdot \tilde{\mu}_{j}\right)=0, m \in 0 . . M,
$$

Denote by $\left\{z_{j}\right\} \cap\left\{\tilde{z}_{j}\right\}$ the intersection of sets $\left\{z_{j} \mid j \in 1 . . k\right\}$ and $\left\{\tilde{z}_{j} \mid j \in 1 . . k\right\}$. Now we define the sets of indices $J_{z z}=\left\{j \mid z_{j} \in\left\{z_{j}\right\} \cap\left\{\tilde{z}_{j}\right\}\right\}, J_{\tilde{z} z}=\left\{j \mid \tilde{z}_{j} \in\left\{z_{j}\right\} \cap\left\{\tilde{z}_{j}\right\}\right\}$, $J_{z}=1 . . k \backslash J_{z \tilde{z}}, J_{\tilde{z}}=1 . . k \backslash J_{\tilde{z} z}$.

Let $s=\left|J_{z \tilde{z}}\right|=\left|J_{\tilde{z} z}\right| \leq k$. Without loss of generality, we assume that $J_{z \tilde{z}}=J_{\tilde{z} z}=1 . . s$ ( $s>0)$, and $J_{z \bar{z}}=J_{\tilde{z} z}=\varnothing(s=0)$. It is clear that in this case $J_{z}=J_{\tilde{z}}=s+1 . . k($ $s<k)$ and $J_{z}=J_{\tilde{z}}=\varnothing(s=k)$.

Denote by $h: J_{z \tilde{z}} \rightarrow J_{\tilde{z} z}$ the total bijection that takes each $j_{1}$ to $j_{2}$ in accordance with the rule $z_{j_{1}}=\tilde{z}_{j_{2}}$. Then the system of equalities (11) takes the form 


$$
\begin{aligned}
& \sum_{j \in J_{z}} z_{j}^{m} \cdot \mu_{j}-\sum_{j \in J_{\tilde{z}}} \tilde{z}_{j}^{m} \cdot \tilde{\mu}_{j}- \\
& -\sum_{j \in J_{z \cap 乏}}\left(\mu_{j}-\tilde{\mu}_{h(j)}\right) \cdot z_{j}^{m}=0, m \in 0 . . M .
\end{aligned}
$$

Further denote

$$
\begin{aligned}
& \xi_{j}=\left\{\begin{array}{l}
\mu_{j}-\tilde{\mu}_{h(j)}, 1 \leq j \leq s \\
\mu_{j}, \quad s+1 \leq j \leq k \\
-\tilde{\mu}_{h(j-k+s)}, k+1 \leq j \leq 2 k-s
\end{array}\right. \\
& \alpha_{i j}=\left\{\begin{array}{l}
z_{j}^{i-1}, 1 \leq j \leq k, 1 \leq i \leq M+1 \\
\tilde{z}_{h(j-k+s)}^{i-1}, k+1 \leq j \leq 2 k-s, 1 \leq i \leq M+1
\end{array}\right.
\end{aligned}
$$

and rewrite (12) in the form

$$
\sum_{j=1}^{2 k-s} \alpha_{i j} \cdot \xi_{j}=0, i \in 1 . . M+1 \text {. }
$$

We note that the leading principal minors of the matrix $\left(\alpha_{i j}\right)$ are Vandermonde determinants composed by powers of distinct numbers. Thus the rank of this matrix is equal to $\min (M+1,2 k-s)$. Let us consider (13) as a system of linear equations with variables $\xi_{j}$. If $M+1 \geq 2 k-s$, the system (13) can have only the trivial solution. In this case $J_{z}=J_{\tilde{z}}=\varnothing, J_{z \tilde{z}}=J_{\tilde{z} z}=1 . . k$, and for all $j \in 1 . . k$ we obtain

$z_{j}=\tilde{z}_{h(j)}, \mu_{j}=\tilde{\mu}_{h(j)}$.

It's mean that $S \approx \tilde{S}$.

Obviously that $M+1 \geq 2 k-s$ for all $s \geq 0$, if $M+1 \geq 2 k$. Hence, if $M \geq 2 k-1$, then the moment problem (10) can have only one solution. The converse may be proved in such a way as is done in [7].

\section{Moment problem for polyline with a finite number of segments}

Let's consider the unclosed polyline $L^{k} \subset \mathrm{R}^{2}$ with $k$ nodes $\left\{t_{j}=\left(x_{j}, y_{j}\right)\right\}_{j=1}^{k}$, and without self-intersections. We assume that associated nodes $t_{j-1}, t_{j}, t_{j+1}$ does not lie on the same straight line, and each node can belong to no more than two segments of a polyline. So polyline has $k-1$ segments. 
As before, we define the map $z: \mathrm{R}^{2} \rightarrow \mathrm{C}$ in accordance with the rule $z=z(t)=z(x, y)=x+i y$. For each of $k-1$ segments we consider the parameterizations $L_{j}^{k}:[0,1] \rightarrow \mathrm{R}^{2}$ that given by $\tau \mapsto L_{j}^{k}(\tau)=t_{j}+\left(t_{j+1}-t_{j}\right) \cdot \tau$.

Let's denote

$$
\begin{aligned}
& z_{j}=z\left(t_{j}\right)=z\left(x_{j}, y_{j}\right)=x_{j}+i y_{j}, \\
& z_{j}\left(\tau_{j}\right)=z\left(L_{j}^{k}(\tau)\right)=z_{j}+\left(z_{j+1}-z_{j}\right) \cdot \tau, \\
& \varphi_{j}=\arg \left(z_{j+1}-z_{j}\right),
\end{aligned}
$$

and take a system of complex moments $S_{m}$ with $m \in 0 . . M \subset \mathrm{N}_{0}$

$$
S_{m}=\int_{L^{k}} z^{m} d L^{k}=\sum_{j=1}^{k-1} \int_{0}^{1} z_{j}^{m}(\tau)\left|d z_{j}(\tau)\right|
$$

After simple calculations we obtain

$$
\begin{aligned}
& S_{m}=\frac{1}{m+1} \cdot\left(-e^{-i \varphi_{1}} \cdot z_{1}^{m+1}+\right. \\
& \left.+\sum_{j=1}^{k-1}\left(e^{-i \varphi_{j-1}}-e^{-i \varphi_{j}}\right) \cdot z_{j}^{m+1}+e^{-i \varphi_{k-1}} \cdot z_{k}^{m+1}\right) .
\end{aligned}
$$

Let $s_{m}=m \cdot S_{m-1}, \mu_{1}=-e^{-i \varphi_{1}}, \mu_{k}=e^{-i \varphi_{k-1}}, \mu_{j}=e^{-i \varphi_{j-1}}-e^{-i \varphi_{j}}$ with $j \in 2 . . k-1$. It's easy that

$$
\sum_{j=1}^{k} \mu_{j}=-e^{-i \varphi_{1}}+\sum_{j=2}^{k-1}\left(e^{-i \varphi_{j-1}}-e^{-i \varphi_{j}}\right)+e^{-i \varphi_{k-1}}=0,
$$

and therefore (15) can be written as (10).

By assumption of polyline we have

$$
\begin{aligned}
& \mu_{1}=-e^{-i \varphi_{1}}=-\frac{\left|z_{2}-z_{1}\right|}{z_{2}-z_{1}} \neq 0, \\
& \mu_{k}=e^{-i \varphi_{k-1}}=\frac{\left|z_{k}-z_{k-1}\right|}{z_{k}-z_{k-1}} \neq 0 . \\
& \mu_{j}=e^{-i \varphi_{j-1}}-e^{-i \varphi_{j}}=\frac{\left|z_{j}-z_{j-1}\right|}{z_{j}-z_{j-1}}-\frac{\left|z_{j+1}-z_{j}\right|}{z_{j+1}-z_{j}} \neq 0,
\end{aligned}
$$

with $j \in 2 . . k-1$.

From the results of the previous section it follows that no exists more than one sets of points $\left\{z_{j}\right\}_{j=1}^{k}$ and the weights $\left\{\mu_{j}\right\}_{j=1}^{k}$ that satisfy (14) with $m \in 0 . .2 k$.

It is easy to prove that the pairs $\left\{\left(z_{j}, \mu_{j}\right)\right\}_{j=1}^{k}$ completely determine the nodes and segments of the polyline $L^{k}$. Indeed, $\mu_{1}$ uniquely determines the value of $e^{i \varphi_{1}}=-\mu_{1}^{-1}$ and 
thus the direction $w=\left(-\operatorname{Re}\left(\mu_{1}^{-1}\right),-\operatorname{Im}\left(\mu_{1}^{-1}\right)\right)$ from node $t_{1}=\left(\operatorname{Re}\left(z_{1}\right), \operatorname{Im}\left(z_{1}\right)\right)$ to the node $t_{2}$. Thus $t_{2}=t_{1}+r \cdot w$ for some real value $r>0$. At least one such point must exist in the node set $\left\{t_{j}\right\}_{j=1}^{k}$ If there are several nodes that satisfy the relations $t_{j}=t_{1}+r_{j} \cdot w$ with some $r_{j}>0$, then we should choose the one which corresponds to the minimum value of $r_{i}$. This follows from our assumption that the points $t_{j-1}, t_{j}$, $t_{j+1}$ should not lie on the same straight line and each node can belong to no more than two segments of a polyline. The same applies for $\mu_{k}$, which together with $z_{k}$ uniquely identifies the segment in the polyline $\left(t_{k-1}, t_{k}\right)$. As for the other pairs of values, we can use the obvious equality $e^{-i \varphi_{j}}=-\sum_{s=1}^{j} \mu_{s}=\sum_{s=0}^{n-j} \mu_{n-s}$ and consistently hold the previous arguments, starting with one of the node $t_{1}$ or $t_{k}$.

A similar result holds for the closed polyline $L^{k}$ defined by the nodes $\left\{t_{j}=\left(x_{j}, y_{j}\right)\right\}_{j=1}^{k}$ with $t_{1}=t_{k}$. In this case, the analogue of (15) takes the form (10) if we substitute $k-1$ for $k$ and put $\mu_{1}=e^{-i \varphi_{k-1}}-e^{-i \varphi_{1}}$.

\section{Applications to the recognition of similarity for planar contours}

A moment problem has a relation to the image analysis [8], including analysis of similarity discrete planar contours [6, 9].

We say that two sets $M_{1} \subseteq \mathrm{C}$ and $M_{2} \subseteq \mathrm{C}$ are called similar, if there exist $\lambda_{0}, \kappa_{0} \in \mathrm{C}$ such that $M_{2}=\left\{z^{\prime} \mid z^{\prime}=\lambda_{0}+\kappa_{0} \cdot z, z \in M_{1}\right\}$. Here $\lambda_{0}$ describes parallel transport of points $M_{1},\left|\kappa_{0}\right|$ is equal to the coefficient of similarity, and $\arg \left(\kappa_{0}\right)$ corresponds to the angle of rotation $\left(\kappa_{0} \neq 0\right)$.

If the similar sets $M_{1}, M_{2}$ are finite, then

$z_{j}^{\prime}-\frac{1}{k} \sum_{s=1}^{k} z_{s}^{\prime}=\lambda_{0}+\kappa_{0} \cdot z_{j}-\frac{1}{k} \sum_{s=1}^{k}\left(\lambda_{0}+\kappa_{0} \cdot z_{s}\right)=$

$\kappa_{0} \cdot\left(z_{j}-\frac{1}{k} \sum_{s=1}^{k} z_{s}\right)$,

and so without loss of generality, we assume that $\sum_{j=1}^{k} z_{j}=\sum_{j=1}^{k} z_{j}^{\prime}=0$ or the same $\lambda_{0}=0$. 
Let's consider two unclosed polyline $L^{k}, L^{\prime k} \subset \mathrm{R}^{2}$ with the same number of segments. As follows from the previous section, each of them is uniquely determined by a system of moments

$$
\begin{aligned}
& \sum_{j=1}^{k} z_{j}^{m} \cdot \mu_{j}=\sum_{j=1}^{k} z_{j}^{m} \cdot \mu\left(z_{j-1}, z_{j}, z_{j+1}\right)=s_{m}, \\
& \sum_{j=1}^{k} z_{j}^{\prime m} \cdot \mu_{j}^{\prime}=\sum_{j=1}^{k} z_{j}^{\prime m} \cdot \mu\left(z_{j-1}^{\prime}, z_{j}^{\prime}, z_{j+1}^{\prime}\right)=s_{m}^{\prime},
\end{aligned}
$$

with $m \in 0 . .2 k-1$ and $\mu\left(z_{j-1}, z_{j}, z_{j+1}\right)$ that satisfy (16)-(18)

Given that

$$
\mu\left(\kappa_{0} \cdot z_{j-1}, \kappa_{0} \cdot z_{j}, \kappa_{0} \cdot z_{j+1}\right)=\frac{\left|\kappa_{0}\right|}{\kappa_{0}} \mu\left(z_{j-1}, z_{j}, z_{j+1}\right),
$$

we finally obtain the similarity criterion for polyline $L^{k}, L^{\prime k}$ in the following form

$$
s_{m}^{\prime}=\left|\kappa_{0}\right| \cdot \kappa_{0}^{m-1} \cdot s_{m}, m \in 0 . .2 k-1 \text {. }
$$

Criterion (21) also holds for closed polylines.

\section{References}

1. Kantorovich LV, Akilov GP. Functional analysis. Moscow: "Nauka" Publisher; 1984. [In Russian]

2. Lavrentiev MM, Saveliev LYa Operator theory and ill posed problems. Novosibirsk: "Institut Matematiki" Publisher; 2010. [In Russian]

3. Khakhlyutin VP. On a problem of integral geometry in the plane (English. Russian original) Sov. Math., Dokl., 1992; 44(2): 574-576; translation from Dokl. Akad. Nauk SSSR, 1991; 320(4): 832-834.

4. Akhiezer NI. The classical moment problem: And some related questions in analysis. Moscow: "Fizmatlit" Publisher, 1961. [In Russian]

5. Edwards RE. Functional analysis. N.Y.: Holt,-Rinehart \& Winston, 1965.

6. Volostnikov VG, Kishkin SA, Kotova SP. Contour analysis and modern optics of Gaussian beams. Computer Optics, 2014; 38(3): 476-481. [In Russian]

7. Gantmacher FR. The Theory of Matrices. Moscow: "Nauka" Publisher, 1960. [In Russian]

8. Pratt WK. Digital image processing. N.Y.: John Wiley \& Sons, Inc, 1978.

9. Furman YaA, Krevetsky AV, Peredreev AK, Rojentsov AA, Khafizov RG, Egoshina IL, Leukhin AN. Introduction into a contour analysis. Ed by Furman YaA. Moscow: "Fizmatlit" Publisher, 2003. [In Russian] 